\title{
Evaluation of angiosuppressive activity of Pleurotus florida on developing chick embryo using chorioallantoic membrane assay
}

\author{
Zacarias $\mathrm{RC}^{1}$, Kalaw $\mathrm{SP}^{1,2}$ and De Leon $\mathrm{AM}^{1,2^{*}}$
}

${ }^{1}$ Department of Biological Sciences, College of Science, Central Luzon State University, Science City of Muñoz, Nueva Ecija, Philippines

${ }^{2}$ Center for Tropical Mushroom Research and Development, College of Science, Central Luzon State University, Science City of Muñoz, Nueva Ecija, Philippines

Zacarias RC, Kalaw SP, De Leon AM 2021 - Evaluation of angiosuppressive activity of Pleurotus florida on developing chick embryo using chorioallantoic membrane assay. Studies in Fungi 6(1), 334-341, Doi 10.5943/sif/6/1/24

\begin{abstract}
Angiogenesis is the proliferation of new blood vessels that enable the normal embryonic development and may also induce growth of numerous pathologies like tumor growth and cancer metastasis. The assays were used for both pro and anti-angiogenic agents. This study evaluated the angiosuppressive activity of Pleurotus florida on the developing chick embryo using chorioallantoic membrane (CAM) assay and used destructive sampling into four different treatment concentrations and a control. The result revealed a decrease in the number of blood vessels branch points formed after 24, 48, and 72 hours at higher treatment concentrations $(0.50 \%$ and $1 \%)$. Statistical analysis showed that 24 hours and 48 hours of exposure to various concentrations have no significant difference. Nevertheless, after 72 hours of exposure, the mean blood vessel branch points formed showed a significant difference among the treatment means between the highest concentration of $P$. florida $(1 \%)$ and the control treatment (sterile water). In terms of the percentage of angiosuppressive activity of $P$. florida with the greatest angiogenic effect, which was the $1 \% P$. florida treatment concentration possesses $35.69 \%$ and $36.85 \%$ angiosuppressive rate after 48 and 72 hours of exposure respectively. Thus, this study showed that P. florida lyophilized hot water extract exhibits angiosuppressive activity on the developing chick embryo using chorioallantoic membrane assay and is concentration and time exposure dependent.
\end{abstract}

Keywords - angiogenesis - blood vessels - destructive sampling - embryonic development pathologies

\section{Introduction}

Angiogenesis is a complex activation, proliferation, and directed migration of endothelial cells to form capillaries from existing blood vessels (Oklu et al. 2010). In human physiology, it plays critical roles ranging from reproduction and fetal growth to wound healing and tissue repair and the sophisticated multi-process of angiogenesis (Yoo \& Kwon 2013). It is vital for normal developmental processes, however may also cause growth of numerous pathologies like tumor growth and metastasis to inflammation and ocular diseases (Auerbach et al. 2003). This means that the formation and differentiation of blood vessels enable normal embryonic development and/or growth of various diseases like tumor and cancer. 
The chorioallantoic membrane (CAM) assay is a well-established and widely used model to examine angiogenesis and anti-angiogenesis. As stated by Nowak-Sliwinska et al. (2014), the chicken chorioallantoic membrane is a highly vascularized extraembryonic membrane, which performs multiple functions during embryonic development, including but not restricted to gas exchange, and its tissue composition and accessibility of the CAM for experimental manipulation, makes it an attractive preclinical in vivo model for drug screening and studies of vascular growth. Avian chorioallantoic membrane is a valuable and convenient model to study angiogenesis and its regulation in vivo. The transparency of the CAM is well-suited for imaging, and the embryo is cultured readily in a petri dish (Tufan \& Satiroglu-Tufan 2005, Parsons-Wingerter et al. 1998).

The oyster mushroom (Pleurotus spp) is highly nutritious, edible mushroom common species in tropical West Africa and Southern parts of Asia (Chang 1991). They are the main mushroom species cultivated in Cameroon ( $P$. ostreatus, $P$. pulmonarius, $P$. florida and $P$. sajor-caju) (Yongabi et al. 2004), where mostly are consumed fresh after cooking, or dried and used in the preparation of stews and soups as substitute for fish or meat (Van et al. 2003). Oyster mushrooms have demonstrated immune-regulatory (Yu et al. 2009), antioxidant (Thillaimaharani et al. 2013, Ramkumar et al. 2010), and anti-inflammatory (Im et al. 2014, Bobek et al. 2001) properties. These beneficial effects are due to their water-soluble polysaccharide component ( $\beta$-glucans), and their phytochemical composition (Fombang et al. 2016).

This research focused on the evaluation of angiosuppressive activity of $P$. florida extracts using CAM assay in developing chick embryos as an animal model in this study if it has a pro or anti-angiogenic effect in the growth of new blood vessels. Moreover, the results that were drawn in this study that illustrate the potential of mushroom extract in medical application may eventually become a baseline information in creating a medical drug in treating diseases including tumor, cancer and other possible attributes.

\section{Materials \& methods}

\section{Hot water extraction of $\boldsymbol{P}$. florida}

Twenty grams air-dried and pulverized $P$. florida was extracted using $600 \mathrm{ml}$ distilled water and undergone $80-90^{\circ} \mathrm{C}$ water bath for 2 hours. The extract was filtered using a Whatman filter paper no. 2 to obtain the aqueous bioactive components of the sample. The filtrates were placed in amber bottles, then lyophilized to dryness at the Department of Rice Chemistry, Philippine Rice Research Institute, Maligaya, Science City of Munoz, Nueva Ecija.

\section{Preparation of experimental treatments and concentrations}

P. florida lyophilized extracts were diluted with distilled water. Concentrations including $1 \%$, $0.5 \%, 0.1 \%, 0.05 \%$ and control (sterile water) were prepared and used as treatments for CAM assay using developing chick embryos. Four treatments with different concentrations of each $P$. florida lyophilized hot water extracts and sterile water as control treatment were prepared in the study. A $10 \mathrm{ml}$ volume of combined lyophilized hot water extract of $P$. florida and sterile water in different concentrations were prepared for each treatment. Table 1 showed the formulation of different concentrations of the experimental treatments.

Table 1 Formulation of different treatment concentrations of $P$. florida lyophilized hot water extract

\begin{tabular}{lll}
\hline Concentrations (\%) & Lyophilized hot water extract $(\mathrm{g})$ & Sterile water $(\mathbf{m l})$ \\
\hline 1.00 & 0.10 & 10 \\
0.50 & 0.05 & 10 \\
0.10 & 0.01 & 10 \\
0.05 & 0.005 & 10 \\
Control & 0.00 & 10 \\
\hline
\end{tabular}




\section{Chorioallantoic membrane assay}

Following the protocol established by Chen et al. (2013) with minor modifications to suit the study, one-day fertilized chick embryos $(65 \pm 6 \mathrm{~g})$ collected from San Pedro East, Rosales, Pangasinan were cleaned with $70 \%$ ethyl alcohol and further pre-incubated at $37.5^{\circ} \mathrm{C}$ in $70-80 \%$ humidity for two days. Egg morphology appears like a meta-ellipse, with a relatively larger side and a smaller one, and the air sac is usually located on the larger side right behind the shell. After disinfection of the shell center outside the air sac, eggs were placed inside a chamber, and a window with $1 \mathrm{~cm}$ by $1 \mathrm{~cm}$ size was made gently over the air sac to break the shell and the vascular zone can be easily identified on the CAM. Sterile paper discs made from punched filter paper were soaked in different concentrations and were directly applied into the vascular zone of the embryo followed by sealing the opening of the egg/embryo and was further incubated for 24, 48 and 72 hours in which three replicates in each concentration was done. After incubation, each CAM was placed in a petri plate, viewed under a stereomicroscope, photographed and quantified by counting the number of blood vessel branch points. The formula used to address the percentage angiosuppressive activity is shown:

$$
\% \text { angiosuppressive }=\frac{\text { initial no. of blood vessel branch points }- \text { total no. of blood vessel branch points formed }}{\text { initial no. of blood vessel branch points formed }} \times 100
$$

\section{Statistical analysis}

Experimental units were laid out following the Completely Randomized Design (CRD). Data that were collected were analyzed using Analysis of Variance (ANOVA) at 5\% level of significance. Also, a Duncan Multiple Range Test (DMRT) to determine which among the treatment means has a significant difference at 5\% level of significance and simple linear regression was performed to quantify the percentage of angiosuppressive activity based on the concentration level.

\section{Results}

\section{Angiosuppressive activity}

Angiosuppressive activity of $P$. florida lyophilized hot water extract using chorioallantoic membrane assay in developing chick embryos as an animal model were evaluated and determined if $P$. florida possesses a pro or anti-angiogenic effect. Three-day fertilized chick embryos were used and exposed to varying treatment concentrations of $P$. florida lyophilized hot water extract and examined after further incubation of 24,48 , and $72 \mathrm{hrs}$ of post treatment application. Table 2 shows the mean number of blood vessel branch points formed in the developing chick embryo treated with P. florida lyophilized hot water extract after 24,48 , and 72 hours of exposure to different concentrations.

Results showed that concentrations have the ability to decrease the formation of blood vessel branch points at 24 hours, 48 hours and 72 hours of exposure to different concentrations and is considered to have an angiosuppressive activity. The lowest number of blood vessel branch points formed was observed in $1 \%$ concentration with a mean number of 24.80 during 24 hours, 36.13 after 48 hours and 41.07 at 72 hours of exposure. On the other hand, the highest number of blood vessel branch points formed was observed in the control treatment (sterile water) with a mean of 42.47 during 24 hours, 56.13 at 48 hours, and after 72 hours of exposure it was recorded to be 66.93.

The mean number of blood vessel branch points formed decreases as the treatment concentration increases in each hour of incubation in comparison with the control treatment, the sterile water which have a mean blood vessel branch points formation of 42.47, 56.13, and 66.33 after 24 hours, 48 hours and 72 hours of exposure to different treatment concentrations respectively. 
Table 2 Number of blood vessels branch points formed in the developing chick embryos treated with $P$. florida lyophilized hot water extract

\begin{tabular}{lccc}
\hline \multirow{2}{*}{ Concentrations (\%) } & \multicolumn{3}{c}{ Mean blood vessel branch } \\
\cline { 2 - 4 } & \multicolumn{1}{c}{$\mathbf{2 4 H R S}$} & \multicolumn{4}{c}{$\mathbf{4 8 H R S}$} & $\mathbf{7 2 H R S}$ \\
\hline 1.00 & $24.80 \pm 3.14^{\mathrm{a}}$ & $36.13 \pm 9.58^{\mathrm{a}}$ & $41.07 \pm 12.62^{\mathrm{b}}$ \\
0.50 & $28.73 \pm 2.91^{\mathrm{a}}$ & $40.73 \pm 7.40^{\mathrm{a}}$ & $48.87 \pm 5.14^{\mathrm{ab}}$ \\
0.10 & $31.60 \pm 2.96^{\mathrm{a}}$ & $41.93 \pm 3.52^{\mathrm{a}}$ & $52.67 \pm 10.23^{\mathrm{ab}}$ \\
0.05 & $37.27 \pm 10.37^{\mathrm{a}}$ & $42.60 \pm 11.33^{\mathrm{a}}$ & $59.80 \pm 3.12^{\mathrm{ab}}$ \\
Control & $42.47 \pm 12.76^{\mathrm{a}}$ & $56.13 \pm 2.08^{\mathrm{a}}$ & $66.93 \pm 7.55^{\mathrm{a}}$ \\
\hline
\end{tabular}

*Values within the parentheses are the standard deviation in each treatment means

*Treatment means with the same letter of superscript are not significantly different from each other at a 5\% level of significance using DMRT

It can be seen in Table 2 that $P$. florida lyophilized hot water extract exhibited an excellent angiosuppressive activity in the highest concentration. However, the mean blood vessel branch points formed during the first two observations which were 24 hours and 48 hours of exposure, revealed that mean blood vessel branch points formed at all treatment concentrations have no significant difference, which means that the data recorded in all treatment concentrations were comparable with the control treatment. Nevertheless, after 72 hours of exposure to different treatment concentrations showed that the highest treatment concentration (1\%), showed a significant difference with the control treatment.

As per the percentage angiosuppressive activity, the results drawn in each treatment were independent from each other due to the fact that the study used destructive sampling in which the total number of blood vessel branch points formed and the initial blood vessel branch points formed were considered. Wherein the data gathered during the observation at control treatment were used as the initial blood vessel branch points formed. Table 3 shows the percentage angiosuppressive activity of $P$. florida lyophilized hot water extract during 48 and 72 hours of exposure.

As shown in Table 3, during 48 hours of exposure to different treatment concentration of $P$. florida lyophilized hot water extract on the developing chick embryos, it was recorded that the percentage angiosuppressive activity was $35.69 \%$ for the highest treatment concentration of $1 \%$ while in the lowest treatment concentration of $0.05 \%$ it was observed to be $23.57 \%$. Then after 72 hours of exposure, the percentage angiosuppressive activity in the highest concentration was $36.85 \%$ and $10.07 \%$ in the lowest concentration. Thus, from these results it can be drawn that the percentage angiosuppressive activity of $P$. florida lyophilized hot water extract treated into the developing chick embryo was directly proportional to the treatment concentrations as it revealed that an increase in the treatment concentration also results to an increase in the percentage angiosuppressive activity. However, based on simple linear regression performed to quantify the percentage of angiosuppressive activity on the concentration level. Table 4 shows that although an increase in the treatment concentration results to an increase in the percentage angiosuppressive activity, the regression equations in both time points were not significant which can be attributed to the number of replicates considered in this study.

Table 3 Percentage angiosuppressive activity of $P$. florida lyophilized hot water extract on the developing chick embryos exposed to different treatment concentration

\begin{tabular}{lll}
\hline Concentrations (\%) & \% Angiosuppressive 48HRS & Activity 72HRS \\
\hline 1.00 & $35.69 \pm 16.43$ & $36.85 \pm 24.26$ \\
0.50 & $27.12 \pm 15.56$ & $25.88 \pm 14.94$ \\
0.10 & $25.33 \pm 4.77$ & $19.69 \pm 22.44$ \\
0.05 & $23.57 \pm 22.99$ & $10.07 \pm 9.17$ \\
Control & 0 & 0 \\
\hline
\end{tabular}


Table 4 Summary of simple regression analysis on the effect of concentration on the percentage angiosuppressive activity by time point

\begin{tabular}{lllllll}
\hline Model & Time & & B & Std. Error & t & Sig. \\
\hline \multirow{4}{*}{1} & & (Constant) & 23.563 & 9.413 & 2.503 & .037 \\
& \multirow{2}{*}{48} & concentration_0.1 & 1.770 & 13.312 & 0.133 & .898 \\
& & concentration_0.5 & 3.560 & 13.312 & 0.267 & .796 \\
& & concentration_1 & 12.120 & 13.312 & 0.910 & .389 \\
\hline \multirow{4}{*}{2} & \multirow{3}{*}{72} & (Constant) & 10.067 & 10.799 & 0.932 & .379 \\
& & concentration_0.1 & 9.623 & 15.272 & 0.630 & .546 \\
& & concentration_0.5 & 15.810 & 15.272 & 1.035 & .331 \\
& & concentration_1 & 26.777 & 15.272 & 1.753 & .118 \\
\hline
\end{tabular}

This result suggests that $P$. florida lyophilized hot water extract is considered an antiangiogenic agent or has an angiosuppressive activity in terms of its bioactive and myco-chemical constituents. It can be noticed that the angiogenic effect of $P$. florida lyophilized hot water extract was dependent on treatment concentrations and time of exposure. The evident effect was the decrease in the number of blood vessels formed with respect to higher concentrations and longer time of exposure (Fig. 1).

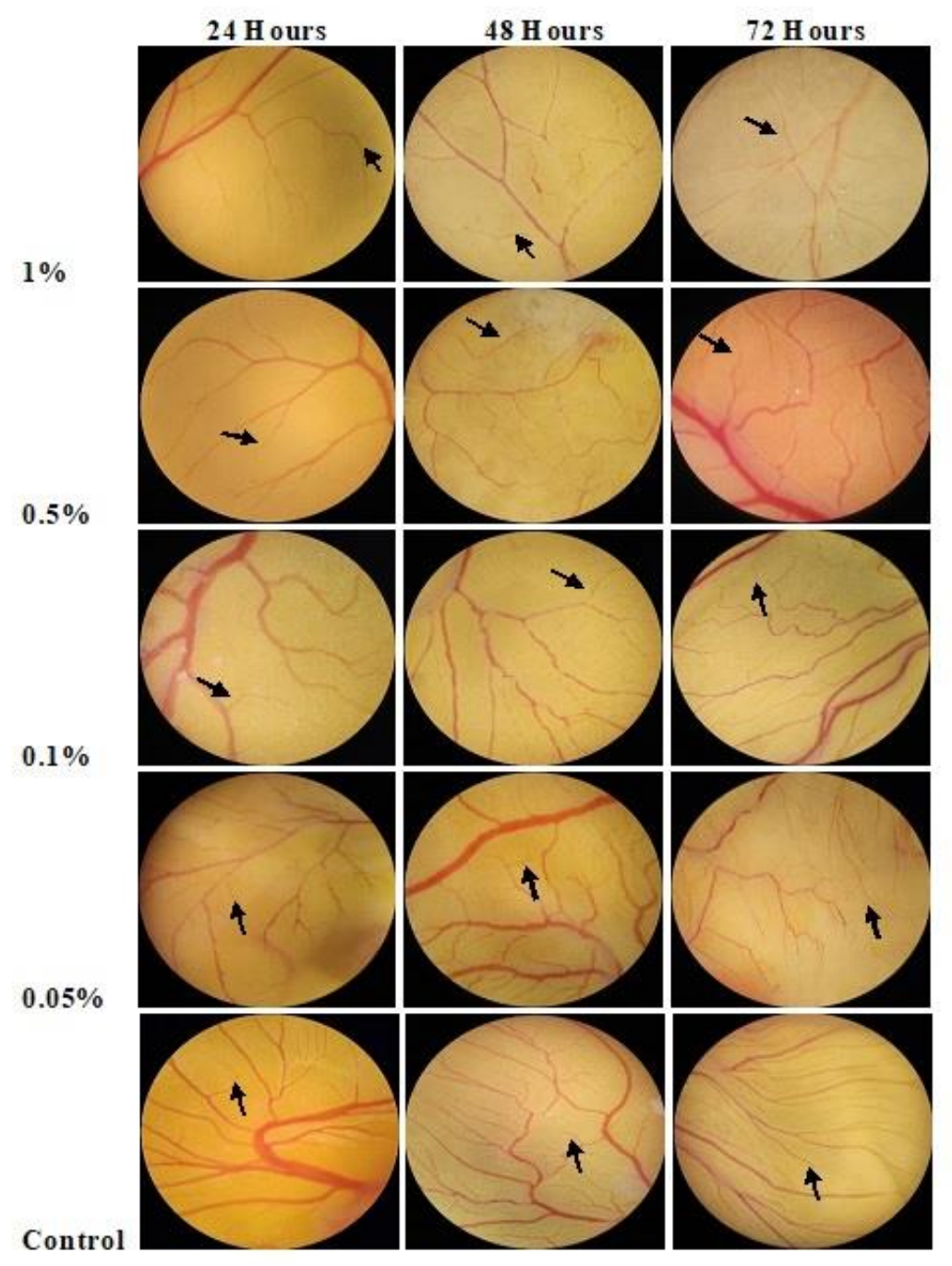

Fig. 1 - CAM assay in chick embryos exposed to various treatment concentrations showing branching points of blood vessels at different time of exposure (arrows showing the blood vessels branch point) 


\section{Discussion}

In the study conducted, agaric macrofungi like $P$. florida lyophilized hot water extract was used to prepare treatment concentrations and were tested to developing chick embryo using CAM assay. The angiosuppressive activity of $P$. florida may be attributed to its antioxidant properties or constituents of mushrooms. A study by Badalyan (2014) revealed that basidiomycetes mushrooms including agaric and bracket fungi are considered to develop different biotech-product due to its bioactive molecules and valuable enzymes in which the main groups of bioactive molecules produced are polysaccharides, terpernoids, phenols, and lectins with more than 120 therapeutic effects revealed such as immune-modulating, antimicrobial, antiviral, antioxidant, and hypocholesterolemic. As what was reported on the study of Lin et al. (2015), the antioxidant and antiangiogenic effects of mushroom phenolic-rich fractions using an ethyl acetate fraction from the mushroom sclerotium of Pleurotus tuber-regium that was rich in total phenolics of $41.4 \pm 0.67$ $\mathrm{mg} / \mathrm{g}$ extract revealed significant inhibition of blood vessel development in the wild type and transgenic zebrafish embryos using endogenous alkaline phosphate assay and microscopic imaging.

Zetter (1998) concluded that angiogenesis is the recruitment of blood vessels that is an essential component of the metastatic pathway. These vessels provide the principal route by which tumor cells exit the primary tumor site and enter the circulation. Thus, this means that studies regarding angiogenesis can be used for pro and anti-angiogenic agents. Several studies indicate that induction of angiogenesis, enhanced tumor growth, and metastasis are potential dose-dependent and concentration-dependent (Soucy et al. 2003, Tosoc et al. 2016, Damjanović et al. 2016).

Several studies shown various inhibitory activities of different mushrooms and are concentration-dependent (Kim et al. 2004, Jung et al. 2007, Lee et al. 2008, Nkembo et al. 2016). The study conducted by Mansouri et al. (2014), which was the mushroom Ganoderma lucidum extracts containing materials exhibited significant reduction in the number of newly formed vessels and expression of inflammatory cytokines and angiogenic factors production from various cells. Similarly, the anti-inflammatory and related activities of mushroom Phellinus linteus ethanol and butanol extraction showed highest anti-inflammatory activity in inhibiting the mouse ear edema and highest inhibitory activity on the chick embryo chorioallantoic membrane angiogenesis in a dosedependent manner (Kim et al. 2004). A similar study conducted by Song et al. (2009) on P. linteus reported that its fruiting bodies that were extracted with $70 \%$ ethanol at room temperature showed a strong anti-angiogenic activity which was detected using the chick embryo CAM assay. On the other hand, another study regarding the methanol extract of $P$. linteus and its fractions as Lee et al. (2010) explained that there was a significant inhibition of proliferation, migration, and tube formation. Moreover, an in vivo angiogenesis assay using the water extract of Grifola frondosa mushroom using chick chorioallantoic membrane revealed a concentration-dependent inhibition of the vascular endothelial growth factor-induced angiogenesis (Lee et al. 2008).

Based on the results and findings of the present study, it can generally be elucidated that the lyophilized hot water extract of $P$. florida has the potential to be an anti-angiogenic agent as it inhibits the vascularization of blood vessels in the course of treatment to different concentration and considered to be concentration-dependent and exposure-dependent.

\section{Acknowledgements}

Our unending gratitude to the following people who willingly contributed and invested their time and effort in order to accomplish this study: Juluis Ceasar C. Abalos, Mr. and Mrs. Rogelio S. Zacarias Jr., Evelyn C. Sinchioco, Jerry C. Lagman, Pamela H. Maniquiz, Janine D. Nieves, Jeanne Rose C. Lazaga and Pia Louise A. Martinez. Special thanks to Mr. John Closter Olivo for his assistance in the statistical analysis and interpretation of results.

\section{References}

Auerbach R, Lewis R, Shinners B, Kubai L, Akhtar N. 2003 - Angiogenesis assays, A critical overview. Clinical Chemistry 49(1), 32-40. 
Badalyan SM. 2014 - Potential of mushroom bioactive molecules to develop healthcare biotech products. International Conference on Mushroom Biology and Mushroom Products 373-738.

Bobek P, Nosálová V, Cerná S. 2001 - Effect of pleuran (beta-glucan from Pleurotus ostreatus) in diet or drinking fluid on colitis in rats. Nahrung 45, 360-363.

Chang ST. 1991 - Recent trends in world production of cultivated edible mushrooms. Mushroom Journal 503, 15-18.

Chen Z, Wen Z, Bai X. 2013 - In vivo chick chorioallantoic membrane (CAM) angiogenesis assays. Bio-protocol 3(18), 1-5.

Damjanović A, Zdunić G, Šavikin K, Mandić B et al. 2016 - Evaluation of the anti-cancer potential of Mahonia aquifolium extracts via apoptosis and anti-angiogenesis. Bangladesh Journal of Pharmacology 11, 741-749.

Fombang EN, Lobe EE, Mbofung CMF. 2016 - Pleurotus florida aqueous extracts and powder influence lipid profile and suppress weight gain in rats fed high cholesterol diet. Journal of Nutrition \& Food Science 6, 473.

Im KH, Nguyen TK, Shin DOB Lee KR, Lee TS. 2014 - Appraisal of antioxidant and antiinflammatory activities of various extracts from the fruiting bodies of Pleurotus Florida. Molecules 19, 3310-3326.

Jung HJ, Jeo HJ, Lim UJ, Ahn EK et al. 2007 - Anti-angiogenic activity of the methanol extract and its fractions of Ulmus davidiana var. japonica. Journal of Ethnopharmacology 112(2), 406-409.

Kim SH, Song YS, Kim SS, Kim BC et al. 2004 - Anti-inflammatory and related pharmacological activities of the $\mathrm{n}-\mathrm{BuOH}$ subfraction of mushroom Phellinus linteus. Journal of Ethnopharmacology 93(1), 141-146.

Lee JS, Park BC, Ko YJ, Choi HG et al. 2008 - Grifola frondosa (maitake mushroom) water extract inhibits vascular endothelial growth factor-induced angiogenesis through inhibition of reactive oxygen species and extracellular signal-regulated kinase phosphorylation. Journal of Medical Food 11(4), 203-210.

Lee YS, Kim YH, Shin EK, Kim DH et al. 2010 - Anti-angiogenic activity of methanol extract of Phellinus linteus and its fractions. Journal of Ethnopharmacology 112(2). 415-421.

Lin S, Ching LT, Chen J, Cheung PCK. 2015 - Antioxidant and anti-angiogenic effects of mushroom phenolics-rich fractions. Journal of Functional Foods 17, 802-815.

Mansouri K, Mahamed-Khosroushahi L, Rasouli H. 2014 - Antiangiogenic/inflammatory behavior of mushroom Ganoderma lucidum extract could be effective for treatment of corneal neovascularization: a hypothesis. Journal of Reports in Pharmaceutical Sciences 3(1), 14-18.

Nkembo AT, Ntantie E, Salako OO, Amissah F et al. 2016 - The antiangiogenic effects of polyisoprenylated cysteinyl amide inhibitors in HUVEC, chick embryo and zebrafish is dependent on the polyisoprenyl moiety. Oncotarget Journal 7(42), 68194-68205.

Nowak-Sliwinska P, Segura T, Iruela-Arispe ML. 2014 - The chicken chorioallantoic membrane model in biology. Medicine and Bioengineering. Angiogenesis 17(4), 779-804.

Oklu R, Walker TG, Wicky S, Hesketh R. 2010 - Angiogenesis and current antiangiogenic strategies for the treatment of cancer. Journal of Vascular Intervention Radiology. 21(12), 1791-1805.

Parsons-Wingerter P, Lwai B, Yang MC, Elliott KE et al. 1998 - A novel assay of angiogenesis in the quail chorioallantoic membrane, stimulation by bFGF and inhibition by angiostatin according to fractal dimension and grid intersection. Microvascular Research 55, 201-214.

Ramkumar L, Ramanathan T, Thirunavukkarasu P. 2010 - Antioxidant and radical scavenging activity of nine edible mushrooms extract. International Journal of Pharmacy 6, 950-953.

Song YS, Kim SH, Sa JH, Jin C et al. 2009 - Anti-angiogenic, antioxidant and xanthine oxidase inhibition activities of the mushroom Phellinus linteus. Journal of Ethnopharmacology 112(2). 410-414.

Soucy NV, Ihnat MA, Kamat CD, Hess L et al. 2003 - Arsenic stimulates angiogenesis and tumorigenesis in vivo. Toxicological Sciences 76, 271-279. 
Thillaimaharani KA, Sharmila K, Thangaraju P, Karthick M, Kalaiselvam M. 2013 - Studies on antimicrobial and antioxidant properties of oyster mushroom Pleurotus florida. International Journal of Pharmacological Science Research 4, 1540-1545.

Tosoc JSP, Frediles VCP, Canda C, Demayo CG. 2016 - Antiangiogenic, antitoxic and antioxidant properties of methanolic extracts of Caladium bicolor (Aiton) Venten. International Journal of the Bioflux Society 8(1), 10-16.

Tufan AD, Satiroglu-Tufan NL. 2005 - The chick embryo chorioallantoic membrane as a model system for the study of tumor angiogenesis, invasion and development of anti-angiogenic agents. Current Cancer Drug Targets 5(4), 249-267.

Van DH, Onguene NA, Kuyper TW. 2003 - Knowledge and utilization of edible mushrooms by local populations of the rain forest of south Cameroon. American Biology 32, 19-23.

Yongabi K, Agho M, Carrera D. 2004 - Ethnomycological study of wild mushrooms in Cameroon, Central Africa. Micologia Aplicada International 16, 34-36.

Yoo SY, Kwon S. 2013 - Angiogenesis and its therapeutic opportunities. Hindawi Publishing Corporation Mediators of Inflammation 127170, 1-12.

Yu S, Weaver V, Martin K, Cantorna MT. 2009 - The effects of whole mushrooms during inflammation. BMC Immunology 10, 12.

Zetter BR. 1998 - Angiogenesis and tumor matastasis. Annual Review of Medicine 49, 407-420. 